\title{
Location choice for renewable resource extraction with multiple non-cooperative extractors: a spatial Nash equilibrium model and numerical implementation
}

\author{
Erik O. Sterner ${ }^{1}$ - Elizabeth J. Z. Robinson ${ }^{2}$ - H. J. Albers ${ }^{3}$ \\ Received: 8 April 2017 / Accepted: 6 June 2018 / Published online: 20 June 2018 \\ (c) The Author(s) 2018
}

\begin{abstract}
Especially in lower-income countries, the distribution of renewable resources in open access settings often reflects the non-cooperative spatial extraction decisions of many individuals who spread out across a landscape. These individuals recognize tradeoffs between distance to the resource, density, and competition amongst extractors. In this paper we present a game theoretic model that explicitly accommodates such explicitly spatial non-cooperative behavior with respect to the extraction of a stationary renewable natural resource, such as a non-timber forest products or bivalvia (for example, oysters, clams), that is located in a two dimensional landscape. Villagers that have identical labor allocations and preferences are shown to undertake very different extraction pathways in equilibrium. For example, some may extract in more congested patches closer to the village while others may extract in less crowded but more distant patches. For many parameterizations, we find multiple spatial Nash equilibria that differ with respect to the number of villagers at each resource location, whether individual villagers extract from one or multiple locations, and the extent and spatial pattern of resource degradation. In addition to finding equilibria with widely different actions taken by identical extractors, the analysis here demonstrates the impact of simplifying assumptions for spatial decisions on predictions of policy impact, resource distributions, and conflict.
\end{abstract}

Electronic supplementary material The online version of this article (https://doi.org/10.1007/s12076-018 -0215-4) contains supplementary material, which is available to authorized users.

$凶$ Erik O. Sterner

erik.sterner@chalmers.se

1 Physical Resource Theory, Department of Space, Earth and Environment, Chalmers University of Technology, Gothenburg, Sweden

2 School of Agriculture, Policy, and Development, University of Reading, Reading, UK

3 Haub School of Environment and Natural Resources, Department of Economics and Finance, University of Wyoming, Laramie, USA 
Keywords Natural resource extraction - Spatial equilibrium - Optimal pathways . Resource patterns $\cdot$ Spatial game $\cdot$ Non-timber forest products

JEL Classification C63 · C72 · O13 · Q56 · Q57 · Q22 · Q23

\section{Introduction}

Rural people in low and middle-income countries tend to be highly dependent on the natural resource base. Whether these individuals extract from forests, hunt wildlife, or catch fish, they make decisions over where to go to collect resources within a twodimensional landscape. Spatial patterns of resource stocks and extraction affect the production of ecosystem services and economic outcomes. In de facto open access settings such as those often found in forests and fisheries in lower-income countries, these patterns derive from the non-cooperative actions of multiple potential resource extractors. When those resources grow in a patchy landscape, villagers make decisions about the number and specific set of patches from which to extract and the level of extraction intensity in each patch. In making these decisions, villagers consider the travel costs of accessing different patches and their opportunity costs of time, the resource stock in each patch, and the extraction decisions of other villagers.

Our field experiences highlight how villagers consistently discuss their extraction decisions in the context of resource availability in different locations, the travel and distance costs of reaching these locations, and spreading out to avoid competition with other extractors. In Xishuangbanna, China, villagers describe a willingness to travel to particular sites known to be productive sources of mushrooms, but describe fuelwood and wild vegetable extraction as more extensive, with the resource distributed throughout the landscape (Albers and Grinspoon 1997). In Thailand, villagers describe informal agreements between villages that divide Khao Yai National Park into piewedges for (illegal) extraction to avoid conflict between villages from overlapping extraction and the resulting resource degradation (Albers and Grinspoon 1997; Albers and Robinson 2007). In Tanzania, community-based forest managers determine the forest entry points for extraction but villagers report that they spread apart from each other to reduce competition for the resource (personal communication, 2013). These observations suggest that understanding resource patterns and resource use therefore requires modeling how individual extractor spatial decisions aggregate across all extractors in the landscape.

Yet despite the prevalence of non-cooperative spatial resource extraction, many models make simplifying assumptions about extractors' spatial decisions or about the interactions of villagers. As examples, many models assume a representative agent; characterize all extractors as allocating labor the same way across patches and wage labor; or constrain extractors' locations such as to only one location, or along one ray (Robinson et al. 2002, 2008; López-Feldman and Wilen 2008; Albers 2010; Behringer and Upmann 2014). Such assumptions, while reasonable for some resources and behaviors, cannot accommodate situations in which extractors choose different pathways through a landscape, and have the opportunity to extract in several places along their chosen path. Thus these more constrained models cannot explore, 
for example, how moving from a non-cooperative de facto open access landscape to a managed landscape affects extractors differentially; or how the introduction of a protected area within the landscape affects the spatial pattern of resource density outside the protected areas through leakage and thus the aggregate ecosystem services provided by the landscape; or how management policies affect the concentration of extractors in different locations, and thus the potential for greater or lower conflict situations.

The objective of this paper is therefore to develop a flexible model that accommodates these more heterogeneous extractor choices and interactions, to provide greater insights into resource extraction, and to explore the implications of various assumptions that constrain extraction choices. We employ a spatial agent based implementation method to find spatial Nash equilibria resulting from a group of identical extractors, with the same goals, labor endowment and landscape, yet who make individual decisions over pathways through the patchy resource, extraction locations, and intensity of extraction at each location. As such, our paper has similarities with sorting models such as Bayer and Timmins (2005) and Timmins and Murdock’s (2007) spatial Nash bargaining game.

For multiple extractors making spatially explicit choices within a landscape, the results provide new insights into the comparison to previous approaches particularly with respect to variation in extractor choices of extraction pathways, the existence of multiple equilibria, and spatial patterns of resource stocks. This flexible model accommodates the reality that all villagers do not necessarily collect from the same areas of the forest (as found in a number of models in the literature, such as Robinson et al. 2008), nor do they necessarily allocate themselves as would a social planner, but rather may spread out taking account of the spatial landscape and the choices of other villagers. Thus our model has clear implications for, for example, the siting of protected areas in landscapes where people traditionally extract resources, and exclusion from specific areas would affect individual extractors differently. The exploration of our model's results reveals the role of distances between patches in determining the extraction heterogeneity across extractors in equilibrium.

The next section describes the setting, the spatial extraction model, and the solution method. The third section presents and discusses the results of the model for different distance settings, and compares the resulting equilibria with constrained extraction location choices. Section four concludes.

\section{Setting, economic model, and solution method}

\subsection{System description}

The system of interest is representative of a rural setting in a lower-income country, with a renewable natural resource spatially distributed across a two dimensional landscape. The resource is stationary and re-grows, such as is the case for many nontimber forest products (NTFPs). The resource is de facto open access, and villagers living adjacent to the area extract the resource across space. Besides extraction of the resource, the locals have access to a wage generating job market. 


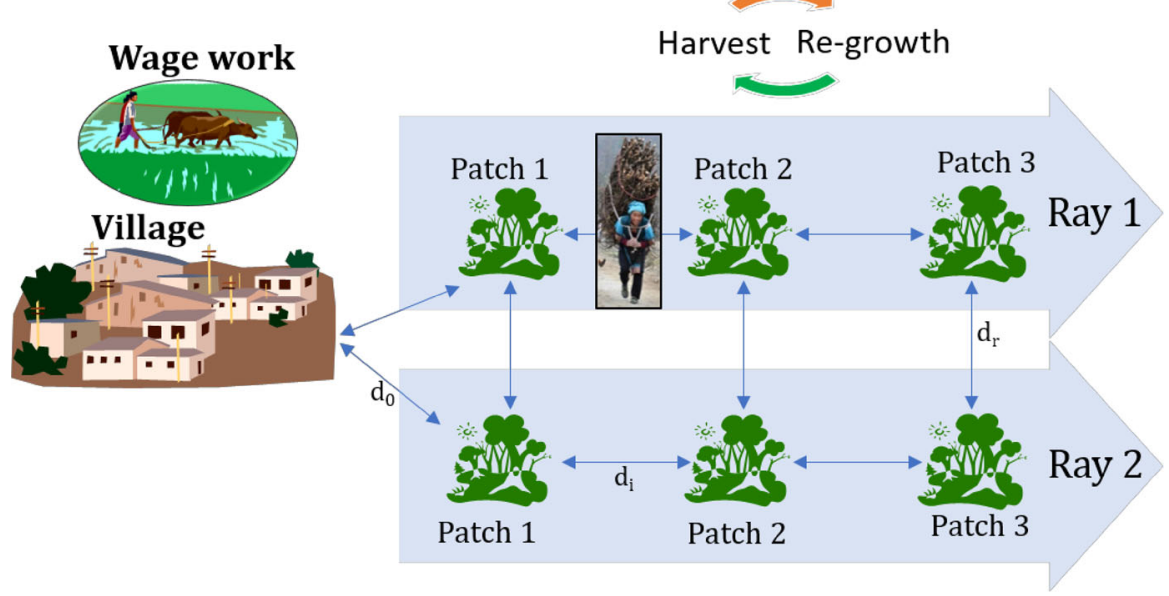

Fig. 1 Illustration of conceptual model developed to study NTFP extraction, displaying the spatial landscape showing village, resource patches, distances between patches, and permissible pathways

\subsection{Conceptual model of villagers and spatial resource setting}

We conceptualize this renewable resource extraction setting to have the following characteristics (Fig. 1):

There are $\mathrm{N}$ villagers and one "village site" or extractor entry point to the de facto open access landscape. A stationary renewable resource is found in discrete "patches," separated by a distance that takes labor time to traverse. The resource extraction is followed by resource re-growth. The spatial setting contains one village located at a distance from a landscape comprising six resource patches arranged in a two by three grid, with three resource patches at increasing distance from the village along each of two "rays" (Fig. 1). Each patch's location is described (r,y) by its ray, $r=1,2$, and its placement at increasing distance from the village on that ray, $y=1,2,3$. We assume that no diagonal travel between rays occurs.

Villagers are identical with respect to labor endowments, effectiveness of their labor whether extracting or working, and preferences. They maximize expected period-byperiod returns to labor because they have no long-term rights to the resource to enable planning. They experience no fatigue from distance traveled or amount of resource being carried. Villagers allocate their labor either to resource extraction (including distance required to get to a particular set of resource patches) or wage work (where the wage is a function of total villager labor supply). Any resource that the villager extracts is sold for an exogenous price at a nearby market. Villagers make their decisions while considering the actions of all other villagers. We solve for the equilibrium resource extraction decisions over both locations and quantities for this group of extractors who face labor allocation trade-offs across distance time costs, extraction, and a nonresource extraction labor option. 


\subsection{Individual extractor's optimization decision}

Each individual, $i$, of $V$ extractors seeks to maximize their individual single period net returns, $W_{i}$, by allocating their labor endowment $L_{i}$ across extraction labor in each patch $(\mathrm{r}, \mathrm{y}), l_{i r y}$; wage labor $l_{i w}$; and distance travel costs $l_{i D}$, which depend on the path taken through the patchy landscape. The travel costs are thus labor costs that reduce the available labor time. The extracted resource is sold at an exogenous price, $p$; and wage labor receives a wage, $w$, which is a function of the total wage labor supply of all extractors. An extractor's labor allocation choices define that extractor's optimal set of patches from which to extract, the pathway for that extraction, and the optimal amount to extract from each patch visited. Each extractor makes decisions based on the labor allocation decisions of all other extractors. We write an individual extractor's optimization as follows:

$$
\max _{L_{i}}\left[W_{i}\left(L_{i}\right)\right]=\max _{\left(l_{i, r, y}, l_{i, w}\right)}\left[p \sum_{r, y} h_{i, r, y}\left(l_{i, r, y}\right)+w_{i}\left(l_{i, w}\right)\right]
$$

s.t.

$$
\begin{aligned}
L_{i} & =\left(\sum_{r, y} l_{i, r, y}\right)+l_{i, w}+l_{i D} \\
H_{r, y} & =q R_{r, y}\left(\sum_{j=1}^{V} l_{j, r, y}\right)^{\gamma} ; \gamma<1 \\
h_{i, r, y} & =\frac{l_{i, r, y}}{\sum_{j=1}^{V} l_{j, r, y}} H_{r, y} \\
w_{i}(l) & =w \cdot \frac{l_{i, w}}{\sum_{j=1}^{V} l_{j, w}} \cdot\left(\sum_{j=1}^{V} l_{j, w}\right)^{\beta} \text { where } \beta<1
\end{aligned}
$$

In Eq. 1, $h_{i, r, y}$ is the amount of the renewable resource extracted by extractor $i$, through the allocation of labor $l_{i, r, y}$ in a particular patch (r, y). The total amount-across all villagers - harvested in any patch, $H_{r, y}$, follows a modified Schaefer-type function that allows for diminishing returns to total labor effort extracting from a particular patch (Zhang and Smith 2011) where $q$ measures an extractability (or catchability) coefficient and $R_{r, y}$ is the resource stock of patch $y$ in ray $r$ at the start of a time period. Thus within a particular time period, the total resource extraction in patch $(\mathrm{r}, \mathrm{y}), H_{r, y}$, is a function of the extraction decisions of all the extractors choosing to extract from that patch. The total harvest each period, $H_{r, y}$, is assumed to be shared in direct proportion to the time each individual extractor spend in a that patch. The wage function is assumed to be endogenous to labor supply with diminishing returns to labor, which is consistent with a lower-income setting. In essence, the wage function shares characteristics with the harvest function but is assumed to have less steep diminishing returns to labor (i.e. $\beta>\gamma$ ). Being a set of optimal labor allocations for all villagers, the model's 
solution comprises individually optimal labor allocation decisions within a spatial Nash equilibrium. Between periods, the resource in each patch partially regenerates after harvest, $\Delta R_{r, y}$, according to a logistic growth function:

$$
\begin{aligned}
\Delta R_{r, y} & =r \cdot\left(R_{r, y}-H_{r, y}\right) \cdot\left(1-\frac{R_{r, y}-H_{r, y}}{K_{r, y}}\right) \\
R_{r, y}^{\prime} & =R_{r, y}-H_{r, y}+\Delta R_{r, y}
\end{aligned}
$$

In Eq. 2, $r$ is the natural growth rate, $K_{r, y}$ is the patch carrying capacity and $R_{r, y}^{\prime}$ is the resource stock after the regrowth.

The sum of the individual labor allocation decisions results in a per-period spatial Nash equilibrium of the labor market and landscape pattern of extraction. Following periods of extraction, the analysis determines the long run socio-ecological steady state (after sufficient time periods such that $R_{r, y}^{\prime} \approx R_{r, y}$ ).

\subsection{Conceptual solution method}

With many individual extractors making complex spatial labor allocation decisions, finding the spatial Nash equilibria requires either simplifying assumptions or computational methods. Because we aim to explore the variety of individual decisions possible, we implement an agent-based method to iterate sequentially through each extractor's choice until an equilibrium is found. Conceptually, in each iteration, an extractor makes a "claim" to a plan (locations and amounts of extraction) in response to the other villagers' claimed plans. The iterations continue with this sequential updating of extractors' plans until no extractor can improve on their plan given the claims of the other villagers, which defines the set of equilibrium plans for all extractors. This mechanism for adapting labor allocation plans is a transparent and realistic mechanism that allows for agents' behavior to be heterogeneous. We base this mechanism on empirical studies such as those reviewed by Bodin and Crona (2009) and our own field experience indicating that villagers in this type of resource extraction setting communicate and generally avoid behaving identically.

This agent based method finds non-cooperative Nash equilibria for different parameter settings without imposing constraints on extractors' choices of pathways and extraction patterns. The resulting set of extraction patterns is the emergent outcome from the interactions of the agents' (here villagers) actions (Bonabeau 2002; Farmer and Foley 2009; An 2012). Our approach differs from those approaches that use equilibrium conditions directly in their solution methods (for example, Robinson et al. 2008), thus relying on tacit assumptions such as that all individuals make identical decisions. In contrast, this model and numerical implementation find equilibria in which extractors identical in labor endowments and preferences can make different extraction labor allocation decisions that maximize their individual returns to labor within a Nash equilibrium setting. 


\subsection{Spatial agent based method to determine the spatial-temporal equilibrium}

To find a per-period Nash equilibrium, we undertake an iterative process of labor allocation decisions chosen by each individual extractor to maximize returns to their labor, given the initial or most recent plans of all other extractors. In order to find the long term spatial-temporal equilibrium, step 7, we iterate the process in steps 1-4 to find the quantity of wage labor and the amount to be extracted (step 5) and the amount of resource re-growth (step 6). The process is as follows:

1. Initial condition. All extractors are assigned an initial plan of their labor allocation across resource patches (with a pre-harvest initial resource density per patch) and wage labor.

2. Extractors are then given an order in which they state their plan.

3. One at a time, each extractor is allowed to adapt her planned labor across patches and wage labor. All extractors know distances to, and current state of, each resource patch, the price of the NTFP, returns to wage labor, and the most recent plans of the other extractors. The extractor calculates her returns to labor in the different patches and to wage labor for all plausible labor allocation alternatives, and chooses the one that would yield the maximum return to her labor. If multiple equally valued alternatives exist, the first one encountered is chosen.

4. When all extractors have stated their plan, the process in step 3 is repeated until no extractor wishes to change her chosen labor allocation. Thus one by one extractors update (i.e. choose to keep or adapt) their plans iteratively, until each has found a labor allocation from which she does not wish to deviate, given the stated labor allocations plans of the other extractors.

5. Single period spatial Nash equilibrium. The end of step 4 implies that a spatial Nash equilibrium has been found and step 5 has extractors act on these final plans at the same time.

6. The period then ends and partial regrowth of the resource follows.

7. Temporal equilibrium or long run steady state. Steps 1-6 are repeated until the resource stocks and the labor allocations show a stable repeating pattern, at which point the long-run steady state for the myopic single period non-cooperative equilibrium has been reached. To identify repeating patterns that do not occur period to period but instead occur cyclically over many periods, the algorithm searches for such cycles to determine multi-year cyclical steady states.

The algorithm is easily adjusted to explore more restrictive non-cooperative equilibria by imposing conditions such as extractors only being able to extract from one patch; extractors only being able to extract from one ray; or all extractors choosing the same extraction pattern to mimic a representative agent assumption.

\subsection{Identifying multiple equilibria}

With many extractors making complex spatial decisions, many different equilibria can arise. To identify many of those equilibria, we evaluate three aspects of the mechanisms for the adaptation of extractors' plans during the iterative process. First, we choose three extremes for the initial condition of assigned labor allocation plans (Step 1 
above): all extractors allocate all their labor to wage labor; all extractors allocate their labor equally across each patch and wage labor; and each extractor is given a randomly assigned extraction pattern. Second, we vary the order in which extractors update their plans between being the same order for each iteration or randomized. Third, we repeat these two variations for different levels of initial resource stocks (i.e. different levels of forest degradation for the initial condition). As with any heuristic algorithm to find approximate solutions to complex optimization problems, these variations in the initial conditions and the ordering of individual choices within the iteration identify many of the multiple equilibria, but cannot guarantee finding all possible equilibria.

We explore the impact of distance on the prevalence of multiple equilibria below. Further sensitivity analysis to study the effects of varying the models parameter values is presented in the supplementary material (SM). We also vary the initial allocation to each extractor for each time period to study its effect on the temporal equilibria. It is varied between using the previous period's allocation as the starting point and assigning a new initial condition per time period.

\subsection{Numerical implementation}

In making a numerical implementation, we introduce discreteness into the labor allocation choice, with the level of resolution reflecting the need for a manageable number of computations. Specifically, extraction in a patch, $l_{i, r, y}$, or wage labor, $l_{i, w}$, is allowed in multiples of $5 \%$ of an extractor's available labor time, after accounting for time spent travelling. Each extractor can visit any number of patches with the constraint that travel time cannot be greater than her total labor endowment. The travel time reflects the shortest travel time costs between patches from which the extractor extracts, assuming diagonal paths are not permitted. This model and solution method can represent highly heterogeneous settings, as we show below in Sect. 3.1. However, in order to understand the basic dynamics of the model, after the first heterogeneous example, we limit the analysis to the study of the symmetrical case with all distances equal, $d_{0}=d_{i},=d_{r}=d$, and demonstrate the impact of distance for three different specific distances between patches, as shown in Sect. 3.2. In the supplementary material we present the model parameter values used in all cases.

\section{Results and discussion}

All villagers are identical with respect to labor endowment and objective function. Yet solutions to the model demonstrate that, in equilibrium, extractors choose different pathways; extract from different patches; may variously extract from one or multiple patches on their chosen path; and some villagers may not extract at all but only engage in wage labor. Extractors spread out to reduce congestion and extract the rents available rather than behaving identically, reflecting what we have observed in practice.

In their sets and pathways of extraction, in equilibrium, individual extractors fall into three general types: patch "specializers" (denoted by $\mathrm{S}_{1}, \mathrm{~S}_{2}$, or $\mathrm{S}_{3}$ in the figures below, depending on whether the individual extracts in the first, second, or third patch in a ray) 
who extract from only one patch; "multipatch"(denoted $\mathrm{MP}_{\mathrm{xy}}$ in the figures) extractors who extract from two or three patches along one ray; and "multiray" (denoted $\mathrm{MR}_{\mathrm{x}, \mathrm{y}}$ in the figures) extractors who extract from patches in both rays. Non-extractors are denoted by "WAGE" in the figures. Which and how many (denoted \#V in the figures) of each type of extractor and non-extractor are found in equilibrium is endogenous to the model parameterization. Finally, depending on the distances between patches, the four different variations of modelling the adaptation of extractors' plans can find multiple equilibria for the same sets of distances. We present subsets or examples of the many Nash equilibria that our solution method finds, and we choose those equilibria that highlight particularly interesting aspects of the model and solution method.

\subsection{Equilibrium patterns of extraction: heterogeneous landscape}

To begin, we explore the results in a policy relevant landscape with heterogeneous forest densities across rays and patches, which reflects the flexibility of this agent based solution method (Fig. 2, parameter details in SM 3.2). We find five distinct villager types in the equilibrium, which are represented visually in the first five panels

\begin{tabular}{|c|c|c|c|c|c|c|c|c|c|}
\hline \multirow{2}{*}{ Wage } & Travel & Wage & Returns & \#V & \multirow{2}{*}{$S_{1}$} & Travel & Wage & Returns & $\# \mathrm{~V}$ \\
\hline & $0 \%$ & $100 \%$ & 0.80 & 1 & & $10 \%$ & $63 \%$ & 0.80 & 1 \\
\hline \multicolumn{10}{|l|}{ Village } \\
\hline \multirow{2}{*}{$\mathbf{S}_{1}$} & Travel & Wage & Returns & \#V & \multirow{2}{*}{$\mathrm{MP}_{12}$} & Travel & Wage & Returns & $\# \mathrm{~V}$ \\
\hline & $10 \%$ & $22 \%$ & 0.88 & 1 & & $20 \%$ & $20 \%$ & 0.81 & 5 \\
\hline \multirow{2}{*}{$\mathrm{MP}_{12}$} & Travel & Wage & Returns & \#V & \multirow{2}{*}{$S_{3}$} & Travel & Wage & Returns & $\# \mathrm{~V}$ \\
\hline & $20 \%$ & $0 \%$ & 0.86 & 3 & & $30 \%$ & $0 \%$ & 0.91 & 3 \\
\hline \multirow{2}{*}{$\mathrm{MP}_{123}$} & Travel & Wage & Returns & $\# \mathrm{~V}$ & \multirow{2}{*}{\multicolumn{5}{|c|}{$\begin{array}{l}\text { Equilibrium Resource Densities } \\
\text { (in \% Carrying Capacity) }\end{array}$}} \\
\hline & $30 \%$ & $0 \%$ & 0.84 & 4 & & & & & \\
\hline
\end{tabular}

Fig. 2 Extraction patterns in heterogeneous landscape. For each villager type-wage only, specializer in patch $i, \mathrm{~S}_{\mathrm{i}}$, or multipatch extractor, $\mathrm{MP}_{\mathrm{iii}}$, - this figure shows the extraction pattern illustrated as a path with the percentage of time spent harvesting in each patch. The number of villagers per type (\#V), and the travel time, wage and returns per type 
of the figure. One villager does not extract, but rather allocates all their time to wage labor (WAGE). Five villagers specialize $\left(S_{\mathrm{X}}\right)$, two in the patches closest to the village and three in one patch furthest from the village. Eight villagers extract from two patches in the same ray $\left(\mathrm{MP}_{\mathrm{xy}}\right)$; and four villagers extract from all three patches in one ray $\left(\mathrm{MP}_{123}\right)$. The aggregation of these individual decisions leads to the heterogeneous resource densities across patches (sixth panel of the figure).

\subsection{Effect of distance on equilibrium patterns: homogeneous initial landscape}

\subsubsection{Intermediate distance $d=0.05$}

With intermediate distances between patches, multiple Nash equilibria arise that comprise many different types of extractors. We highlight two of the equilibria that emerge for $d=0.05$ (Fig. 3) that are fundamentally different. In one, each villager "distance specializes" (Fig. 3a), extracting from patches in one or both rays at the same distance from the village. In the other highlighted equilibrium, villagers "ray specialize" (Fig. 3b), extracting from one or multiple patches in one ray. The equilibrium resource densities after harvest for Equilibrium 1 is 59, 62 and $67 \%$ of the carrying capacity. The corresponding percentages for Equilibrium 2 are 60, 63 and $65 \%$.

In other equilibria for the intermediate distance $d=0.05$, we find a combination of multipatch, multiray, and specializer extractors. In a particular equilibrium, up to four different extractor types emerge who extract in at least one of the four patches closest to the village (SM). This outcome illustrates the considerable heterogeneity in extractors' spatial extraction decisions, as they locate across the landscape to take full advantage of the available rents. Over the various multiple Nash equilibria found for this distance, out of the total of 18 extractors, the number choosing only wage labor varies from 1 to 3 ; the number of extractors only going to one ray varies from 2 to 15 ; and the number of extractors focusing on only one distance into the forest varies from 5 to 16 .

\subsubsection{Large distance: $d=0.15$}

In contrast, when distance costs are sufficiently large, for each distance there is one unique spatial Nash equilibrium in which each villager either extracts from just one patch or only engages in wage labor. For $d=0.15$, there are 13 "specializers" while five villagers only engage in wage labor (see SM Figure SM1). The distance to the patches protect them for this landscape and the level of degradation is low. The equilibrium resource densities after harvest across both rays at increasing distance from the village are 55,66 and $86 \%$ of the carrying capacity. That density profile reflects that distance costs limit extraction time.

\subsubsection{Small distance: $\mathrm{d}=\mathbf{0} 0005$}

When patches are almost contiguous, $d=0.005$, the distance costs of moving between patches is very low and the spatial dimension almost disappears. Many Nash equilibria 
a

\begin{tabular}{||c|c|c|c|c|}
\hline Wage & Travel & Wage & Returns & $\# \mathrm{~V}$ \\
\cline { 2 - 5 } & $0 \%$ & $100 \%$ & 0.82 & 2 \\
\hline $0 \%$ & $0 \%$ \\
\hline Village & $0 \%$ \\
\hline
\end{tabular}
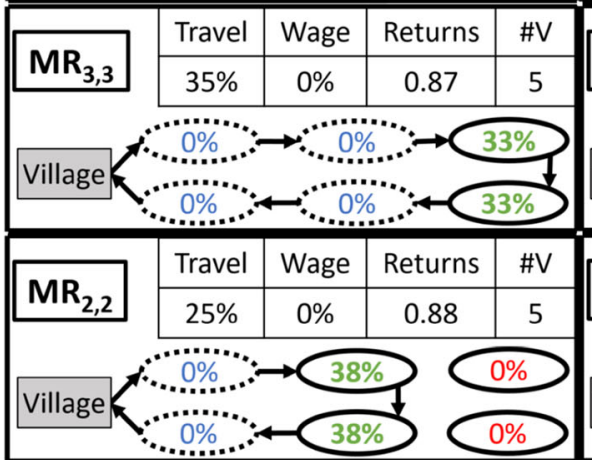

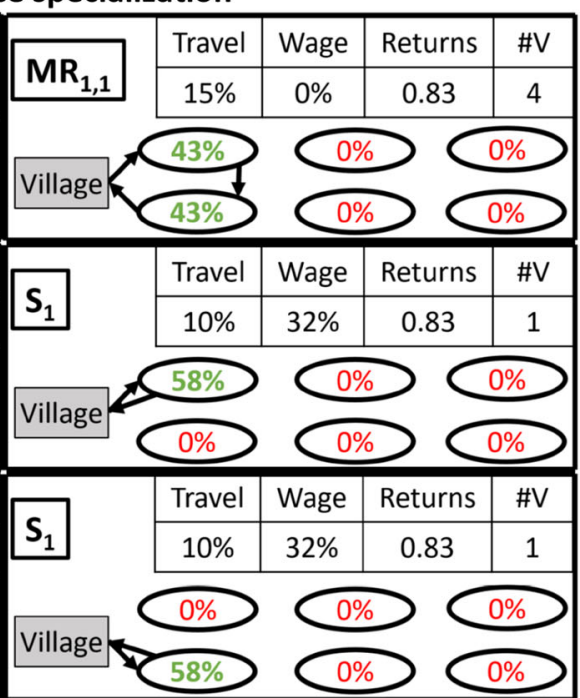

b

Equilibrium 2: Ray specialization

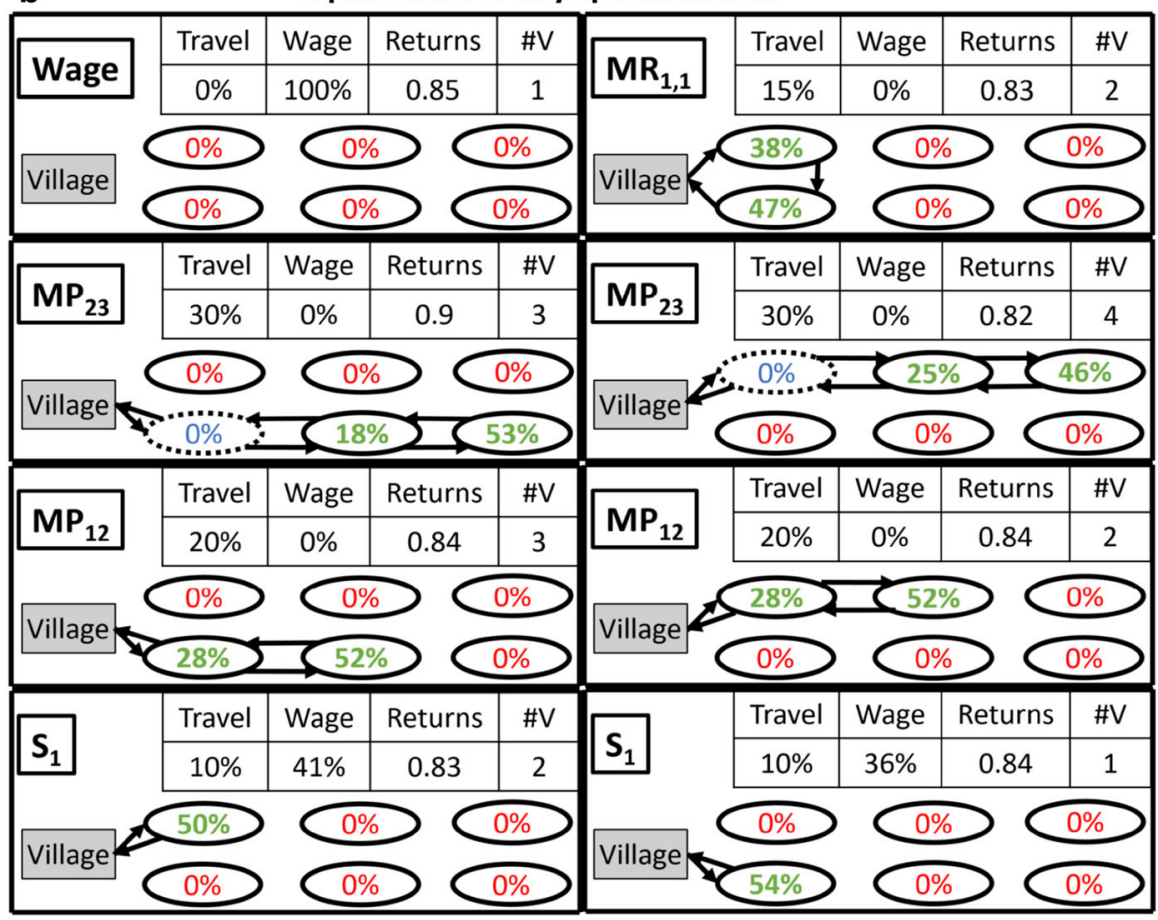

Fig. 3 Extractor types and labor choices for two Nash equilibria for the "Intermediate" distance $(d=0.05)$ scenario. In the first equilibrium (a), the majority of the extractors extract at a certain distance away from the village. The second equilibrium (b) is characterized by a majority of extractors going to only one ray 
are found in which most or all extractors extract from every patch (Multiray 123,123), with varying levels of labor allocated to each patch (see SM Figure SM2). The equilibrium resource densities after harvest for are 50,50 and 51\% of the carrying capacity.

\subsubsection{Sensitivity analysis}

A sensitivity analysis was performed to explore the effect on the results of varying the model parameters (see SM). For the intermediate distance scenario, the individual parameters were varied systematically with all parameters varied at least twice, with one low value and one high value (see SM for more detail). The results from the sensitivity analysis show that the simulation results presented here are robust. The most noteworthy result from the sensitivity analysis was that for a larger value on $\gamma$ (see Eq. 1) that allows for initially less diminishing harvest per extraction time, the tendency of distance specialization is replaced by ray specialization.

\subsection{Discussion of the equilibrium patterns of extractor choices}

A number of clear regularities emerge from our model simulations. First, when patches are close together, individual extractors spread their resource collection across patches and rays, resulting in a large number of extractors in each patch, particularly in the closest patches. When patches are further apart, we find more instances of different types of specialization, some extract at only one distance, some in only one ray, and some in only one patch. At larger distances still, all villagers are wage or patch specializers. Second, we find multiple equilibria when the patches are not too far apart; whereas for larger distances we find a unique Nash equilibrium for each distance. Third, as we parametrically increase distance between the patches, we stop finding multiray extractors at relatively small distances, such as for an intermediate parameterization with $\mathrm{d}=0.075$, whilst we continue to find multipatch extractors (i.e. ray specializers) for larger distances.

The interplay of distance costs moving from one resource patch to another, the spatial inter-connectedness of patches (such that individuals extracting from more distant patches must pass through the closer patches), and diminishing returns to total labor extracting in a particular patch, drives these complex patterns of equilibrium extraction. Individually, extractors face trade-offs between spending labor time travelling to more distant patches, and spending more time extracting from patches they must pass through to reach these distant patches. These choices are more pronounced when patches are further apart. Individuals only extracting from nearer patches cannot do better incurring the greater distance time costs to extract in the more distant patches, whilst villagers extracting from these more distant patches cannot do better by allocating any labor to extraction in the nearer patches. Thus for large distances, we find a unique Nash equilibrium with all villagers specializing, implying that some extractors pass through nearby patches without any extraction on their way to more distant patches (see patches marked 0\% and dotted outline in Figs. 2 and 3); and with resource stocks greater and marginal returns to labor higher with more distant the patch from the village. 
When multiple equilibria exist for a given distance $d$, at least some individuals must extract from more than one patch (i.e. at least some extractors are not patch specializers). Some of these equilibria differ in the set of extractor types that are found. For example, when the solution method's initial labor allocation plan for all villagers is all wage labor, equilibria more frequently have extractors mainly specializing at one distance into the resource landscape but in both rays (see Equilibrium 1 in Fig. 3). In contrast, when the solution method's initial labor allocation plans are random spatial patterns, the equilibria are dominated by ray specializers (see Equilibrium 2 in Fig. 3 ). Other multiple equilibria comprise the same set of extractor types but differ in how extractors split their labor across the same set of patches (and are hence simple expected permutations of villagers' extraction patterns). These multiple equilibria occur when the average returns to labor for wage and extraction in different patches are equal, implying distances between patches do not drive wedges between the marginal returns to labor in these different patches. Further, the total labor extracting in each patch is approximately the same for all equilibria.

Finally, there is a natural asymmetry between moving within a ray, and moving across rays, with the cutoff distance for finding multiray extractors smaller than for finding multipatch extractors. By moving laterally, extractors have access to an additional patch in the alternate ray at a distance time cost that is half of that of moving to the next patch along the same ray, but this patch is more accessible to individuals only extracting within that alternate ray. This kind of tradeoff underpins the occurrence of the two different types of multiple equilibria in which "ray specialization" or "distance specialization" is more frequent among the extractors.

\subsection{Constrained extraction}

To consider the impact of common modeling assumptions, we contrast the long-run equilibrium patterns of resource extraction explored above with equilibria reached if we make various modeling assumptions that restrict the choices extractors can make over the path they take through the landscape and the number of patches that they can extract from.

\subsubsection{One patch restriction}

First, we consider the implications of constraining individuals to extract from one patch (López-Feldman and Wilen 2008, restrict extractors to one location). This restriction only affects extractors in lower distance settings (including our intermediate and small distance examples), because, in high distance cost settings, unconstrained extractors are all patch specializers. We find a unique spatial Nash Equilibrium for each distance. Although this restricted case forces each extractor to harvest in one location, their location decisions are not pre-defined and the results demonstrate spatial heterogeneity in the chosen location.

For the intermediate distance in this one-patch constrained case, we find fewer extractors on average in each patch, with those extracting from patch 3 obtaining returns to their labor almost $20 \%$ greater than for the unconstrained individuals who 


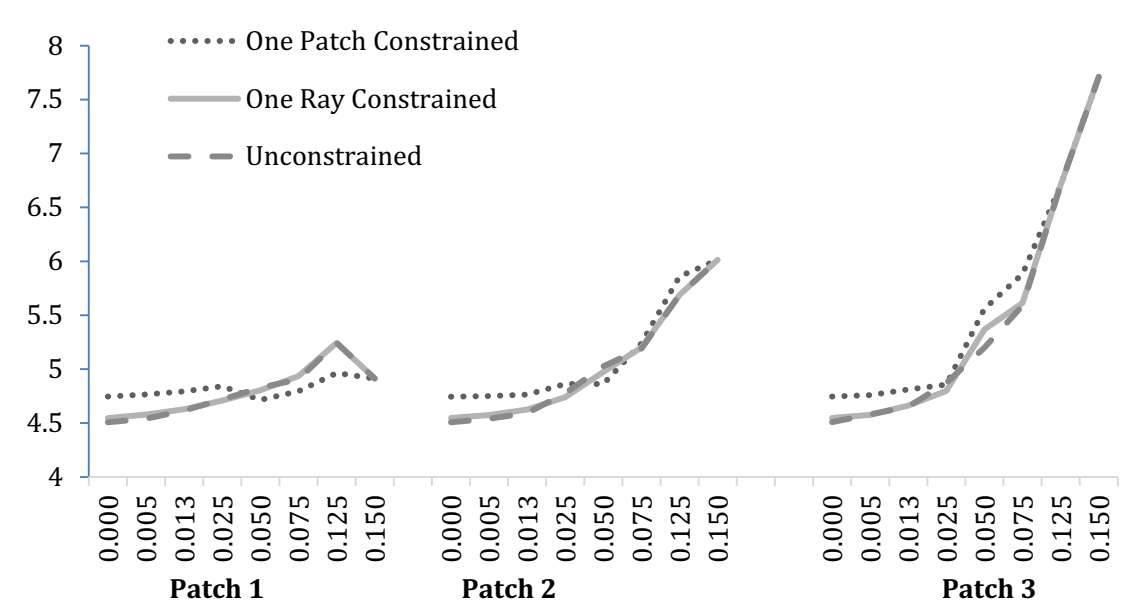

Fig. 4 Impact of constraints on villager choices on average resource stocks

extract from patch 3, but those extracting from closer patches obtaining lower returns (Figure SM4a). The constrained small distance setting produces an equilibrium with 3 extractors in each of the six patches. This pattern is in distinct contrast to the unconstrained model where all 18 individuals extract from the closest patches, and returns to labor are lower. Because one-patch constrained extractors do not have the option to cover their distance costs by extracting in multiple patches along a pathway, for smaller distances, the one-patch constraint in general leads to fewer extractors extracting more from more distant patches, and higher equilibrium levels of resource stocks in nearby patches (Fig. 4). Thus the one-patch constraint mischaracterizes both the extractor behavior and the pattern of resource stocks in any setting with low to moderate distance costs.

\subsubsection{One ray restriction}

Similar to the one patch restriction, a one ray restriction only affects the equilibrium for smaller distances for which at least some unconstrained extractors would choose to extract from more than one ray (including the intermediate and small distance examples above). Many studies, implicitly or explicitly, assume extraction along a line from a village (for example Albers and Robinson 2011). With our model, we still find multiple equilibria in which some extractors are multipatch extractors, and others specializers. However, by construction there are no multiray extractors. Such modelling choices change the spatial patterns of resource extractors, but have minimal impact on the spatial resource stock profile for the studied landscape geometries.

\subsubsection{Representative extractor}

The findings here support Noailly et al. (2003: 184)'s statement that "agents can exhibit heterogeneous characteristics, interactions between which often lead to 'emergent' patterns that cannot be easily predicted at the population level by using representative 
agent models". To characterize the representative agent setting here, we constrain all extractors to follow the same pathway through the landscape and make the same labor choices over extraction. We find that for both small and intermediate distances, extractors enter all six patches, behaving as identical Multiray ${ }_{123,123}$ extractors.

For the small distance $\mathrm{d}=0.005$, compared with the unconstrained case, assuming a representative agent leads to considerably lower extraction per patch and per extractor, and consequently equilibrium stock levels and wage labor are higher. This difference in extraction labor occurs because the representative agent simulates implicitly a rudimentary form of cooperation among identically behaving extractors. For the large distance case, the representative agent allocates all labor to wage labor, in distinct contrast to the unconstrained model.

There are some distances for which the long run equilibrium is cyclical for the representative agent case. For example, for $\mathrm{d}=0.135$, the long run equilibrium comprises a repeating cycle where the representative extractor extracts as a Multiray ${ }_{12,12}$ extractor for two time periods, and then every third period, they only do wage labor. This cyclical equilibrium is characterized by alternating intense and zero extraction over space and over time. Such "pulsing" is found in the fishing literature (Sluczanowski 1984; Nøstbakken 2006; Maroto et al. 2009), in situations where moving between resource patches is costly, or when there is uncertainty, or, as is the case here, where there is a representative agent (as is also the case for Robinson et al. 2008).

\section{Conclusion}

The model and implementation developed here permit exploration of the patterns of resource extraction and resource stocks that derive from the interactions of multiple extractors' spatial labor allocation decisions in a patchy resource setting. Villagers that face the same landscape, objective function, and labor endowment choose very different sets of patches from which to extract, with these choices highly sensitive to the distances between resource patches. Using a spatial agent based method of sequential labor allocation plans that iterates towards a Nash equilibrium, our solution method reveals the spatially heterogeneous extractor behavior. Further, the solutions clearly demonstrate that simplifying assumptions such as representative agents and singlepatch extraction can misrepresent the extraction patterns across the spatial setting and cannot reveal complex patterns of extraction pathways or heterogeneity in choices even from ex ante identical extractors. A representative agent assumption is most likely to misrepresent the extraction patterns of villagers when distances between patches are larger, and underestimate total extraction and thus degradation. A single patch extraction assumption is most likely to misrepresent the extraction patterns of villagers when distances between patches are smaller.

Different model assumptions generally suggest small differences in resource profile and overall stocks, but much greater differences in the number of extractors found in each patch. The unconstrained model typically finds many more individuals extracting in each patch. This outcome has implications for conflict between extractors, and for species that are not extracted or hunted, but are sensitive to human presence. Differences in the returns to individuals are also found for the variously constrained 
model assumptions explored. Such differences in the returns to individuals are much smaller for the unconstrained model. Multiple equilibria are found and exhibit a variety of differences in villagers' extraction pattern that do not affect the overall extraction.

This model and solution method provide the needed flexibility to explore important policy options relevant particularly to a lower-income setting, in contrast to many more simplified or constrained models. For example, our model shows explicitly the extent to which we should expect to find relatively large numbers of villagers extracting in any one area, with the concomitant scope for conflict between these villagers under a de facto open access setting. A natural next step in ongoing work explores how the spatial pattern of extraction is predicted to change if extraction is coordinated, and the extent to which extractors are better off and the resource less degraded. This analysis will allow us to explore whether, and the extent to which, the benefits of management and coordinated extraction outweigh the costs across dimensions of ecosystem services, livelihoods, and conflict. This model is particularly useful for exploring explicitly spatial management of a landscape, in which policy makers can choose to introduced protected zones, managed zones, and open access zones; or alternatively to restrict the total amount extracted and allow extractors to choose from which locations they collect the resource. This paper highlights sensitivity to model assumptions that have implications for estimated conflict, equity, and natural resource management.

Acknowledgements The authors are grateful for funding from the Environment for Development Initiative for fieldwork funding and to the Knobloch Wyoming Excellence endowment for travel support. We thank Kristian Lindgren for valuable discussions.

Open Access This article is distributed under the terms of the Creative Commons Attribution 4.0 International License (http://creativecommons.org/licenses/by/4.0/), which permits unrestricted use, distribution, and reproduction in any medium, provided you give appropriate credit to the original author(s) and the source, provide a link to the Creative Commons license, and indicate if changes were made.

\section{References}

Albers, H.J.: Spatial modeling of extraction and enforcement in developing country protected areas. Resour. Energy Econ. 32, 165-179 (2010)

Albers, H.J., Grinspoon, E.: A comparison of the enforcement of access restrictions between Xishuangbanna Nature Reserve (China) and Khao Yai National Park (Thailand). Environ. Conserv. 24, 351-362 (1997)

Albers, H.J., Robinson, E.J.: Spatial-temporal aspects of cost-benefit analysis for park management: an example from Khao Yai National Park, Thailand. J. For. Econ. 13, 129-150 (2007)

Albers, H.J., Robinson, E.J.: The trees and the bees: using enforcement and income projects to protect forests and rural livelihoods through spatial joint production. Agric. Resour. Econ. Rev. 40, 424 (2011)

An, L.: Modeling human decisions in coupled human and natural systems: review of agent-based models. Ecol. Model. 229, 25-36 (2012). https://doi.org/10.1016/j.ecolmodel.2011.07.010

Bayer, P., Timmins, C.: On the equilibrium properties of locational sorting models. J. Urban Econ. 57, 462-477 (2005)

Behringer, S., Upmann, T.: Optimal harvesting of a spatial renewable resource. J. Econ. Dyn. Control 42, 105-120 (2014)

Bodin, Ö., Crona, B.I.: The role of social networks in natural resource governance: what relational patterns make a difference? Glob. Environ. Change 19, 366-374 (2009). https://doi.org/10.1016/j.gloenvcha. 2009.05.002

Bonabeau, E.: Agent-based modeling: methods and techniques for simulating human systems. Proc. Natl. Acad. Sci. 99, 7280-7287 (2002) 
Farmer, J.D., Foley, D.: The economy needs agent-based modelling. Nature 460, 685-686 (2009)

López-Feldman, A., Wilen, J.E.: Poverty and spatial dimensions of non-timber forest extraction. Environ. Dev. Econ. 13, 621-642 (2008)

Maroto, J.M., Moran, M., Sandal, L.K., Steinshamn, S.I. (2009) Continuous harvesting costs in sole-owner fisheries with increasing marginal returns. In: NHH Department of Finance and Management Science Discussion Paper No. 2009/6. Available at SSRN: https://ssrn.com/abstract=1496316

Noailly, J., van den Bergh, J.C., Withagen, C.A.: Evolution of harvesting strategies: replicator and resource dynamics. J. Evol. Econ. 13(2), 183-200 (2003)

Nøstbakken, L.: Regime switching in a fishery with stochastic stock and price. J. Environ. Econ. Manag. 51(2), 231-241 (2006)

Robinson, E.J.Z., Williams, J.C., Albers, H.J.: The influence of markets and policy on spatial patterns of non-timber forest product extraction. Land Econ. 78, 260-271 (2002)

Robinson, E.J., Albers, H.J., Williams, J.C.: Spatial and temporal modeling of community non-timber forest extraction. J. Environ. Econ. Manag. 56, 234-245 (2008)

Sluczanowski, P.R.: A management oriented model of an abalone fishery whose substocks are subject to pulse fishing. Can. J. Fish. Aquat. Sci. 41(7), 1008-1014 (1984)

Timmins, C., Murdock, J.: A revealed preference approach to the measurement of congestion in travel cost models. J. Environ. Econ. Manag. 53, 230-249 (2007)

Zhang, J., Smith, M.D.: Estimation of a generalized fishery model: a two-stage approach. Rev. Econ. Stat. 93(2), 690-699 (2011) 